\title{
Plataforma e-consumo: Monitoramento Inteligente do Consumo de Energia Elétrica em Residências
}

\author{
Larissa S. Del Rio ${ }^{1}$, Andre R. Eichner ${ }^{1}$, Tiago A. Rizzetti ${ }^{1}$ \\ ${ }^{1}$ Colégio Técnico Industrial de Santa Maria - Universidade Federal de Santa Maria (UFSM) \\ Av. Roraima, 1000 - 97.105-900 - Santa Maria - RS - Brasil \\ \{larissa,eichner\}@redes.ufsm.br, rizzetti@ctism.ufsm.br
}

\begin{abstract}
In Brazil, according to the National Energy Balance for the year of 2016, the residential sector consumes more energy than the service sector. From this, this work has as its objective the development of a platform capable of monitoring consumption of electricity from a residence. The platform will be able to gauge the individual consumption of household equipment with the use of a measuring device and a where the user will be able to view the consumption of each equipment and have an estimate of the amount to be paid. Tests were performed in a real scenario, with the measurement of electricity consumption in three different equipment. With the obtained results it was possible to observe the efficiency of the proposed platform, offering the user transparency with its expenses related to the electric energy.
\end{abstract}

Resumo. No Brasil, segundo o Balanço Energético Nacional referente ao ano de 2016, o setor residencial consome mais energia que o setor de serviços. A partir disso, esse trabalho tem como objetivo o desenvolvimento de uma plataforma capaz de monitorar o consumo de energia elétrica de uma residência. A plataforma será capaz de aferir o consumo individual dos equipamentos da residência com o uso de um dispositivo para realizar a medição, além de possuir uma interface de monitoramento, onde o usuário será capaz de visualizar o consumo de cada equipamento e ter uma estimativa do valor a ser pago. Foram realizados testes em um cenário real, com a medição do consumo de energia elétrica em três equipamentos diferentes. Com os resultados obtidos foi possível observar a eficiência da plataforma proposta, oferecendo ao usuário uma maior transparência com seus gastos relacionados à energia elétrica.

\section{Introdução}

No cenário atual, o termo redes elétricas inteligentes (REIs) encontra-se bastante difundido. O conceito de Smart Grids (SG) pode ser definido como o uso de dispositivos de comunicação inteligentes na rede responsável pelo transporte de energia. A principal função desses dispositivos é o envio de informações para a central de controle da concessionária de energia elétrica. Essas informações são essenciais para a correta operação e monitoramento da rede elétrica, do sistema como um todo [CGEE 2012].

Um dos principais dispositivos utilizados nas SG é o medidor inteligente (smart meter), responsável pela maioria das tarefas da rede inteligente uma vez que este participa de várias aplicações das REIs, seja atuando como produtor de dados ou como elemento de configuração. Entre suas funções encontram-se o processamento de dados e o 
envio de determinados comandos para outros dispositivos. Além disso, faz o envio de notificações para a concessionária quando há falta de energia elétrica, realiza o monitoramento do consumo e da qualidade e é capaz tanto de enviar como de receber informações [Zhou et al. 2017].

A plataforma proposta neste trabalho é composta por um dispositivo inteligente capaz de aferir o consumo de energia elétrica e uma interface web para monitoramento desse consumo. Esse dispositivo é capaz de aferir o consumo e enviar essas informações através da rede de dados local do usuário. Porém, o mesmo não possui qualquer ligação com a concessionária de energia, assim pode ser definida como uma plataforma para controle do consumo residencial.

Nas próximas seções deste artigo estão o embasamento teórico necessário para seu desenvolvimento, a descrição da plataforma desenvolvida, testes e resultados. E, por fim, as conclusões e trabalhos futuros.

\section{Smart Grids}

As Smarts Grids representam a modernização do sistema elétrico que existe atualmente, onde a adição de dispositivos inteligentes provê uma maior eficiência, automatização de tarefas e segurança. Uma SG é um conjunto de dispositivos, aplicações e suas comunicações, que possibilita a criação de um fornecimento de energia elétrica amplamente distribuído e automatizado [Yan et al. 2013]. A Advanced Metering Infrastructure (AMI) é uma das aplicações de SG que possui várias ramificações. Algumas destas são de controle exclusivo da concessionária, como medição geral do consumo de usuário, valor de tarifação, atuando portanto na LAN ou NAN da concessionária. Outras, são centralizadas na HAN, onde encontra-se o foco deste trabalho. Esta última abrange o gerenciamento e consumo individual de cada dispositivo dentro do ambiente do cliente de energia elétrica (consumidor).

\section{Medição Inteligente}

Os medidores inteligentes são dispositivos eletrônicos responsáveis pela maior parte das tarefas existentes em uma SG. Esse é o elemento essencial para implantação da AMI. A medição realizada até o medidor inteligente do cliente é gerida pela concessionária de energia, porém na HAN a situação de gerência dos equipamentos e, também, a comunicação entre os dispositivos é responsabilidade do próprio consumidor.

Esses dispositivos também precisam compartilhar informações de forma segura, seja com o medidor inteligente ou com outros mecanismos como o proposto neste trabalho. Dessa forma, na próxima seção será apresentado o protocolo MQTT que é o padrão de comunicação utilizado na plataforma proposta.

\section{Protocolo MQTT}

O MQTT (Message Queue Telemetry Transport) é um protocolo de mensagens otimizado para utilização em redes não confiáveis e/ou de alta latência, que contém equipamentos com capacidade de processamento e memória bastante limitados. Seu modo de comunicação baseia-se no modelo publicador/subscritor (publish/subscriber).

$\mathrm{O}$ protocolo possui duas entidades principais: o message broker e os clientes. $\mathrm{O}$ broker é o servidor da comunicação, que recebe todas as mensagens dos clientes e as 
redireciona para destinos relevantes. Os clientes são qualquer "coisa" que possa interagir com o broker e receber mensagens [Yuan 2017]. A Figura 1 mostra um exemplo prático do funcionamento do modelo de publicação e assinatura.

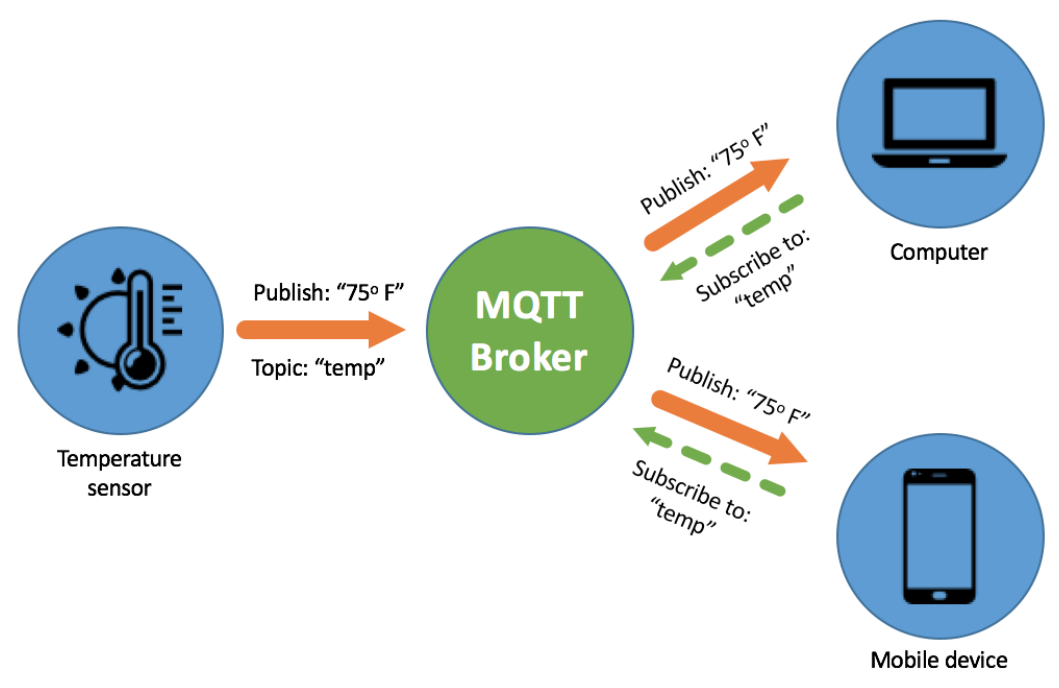

Figura 1. Modelo de Comunicação MQTT.

Conforme pode ser visualizado na Figura 1, o sensor de temperatura publica uma mensagem com a temperatura atual no tópico "temp", ao receber a mensagem o broker encaminha aos clientes Computer e Mobile device, que estão subscritos no mesmo tópico "temp". Na seção seguinte será apresentada a plataforma desenvolvida e as tecnologias que a compõem.

\section{Plataforma e-consumo}

A plataforma proposta nesse trabalho está dividida em duas partes principais: o hardware de baixo custo e a interface web de monitoramento. O hardware é responsável por coletar as informações de corrente elétrica consumida pelos equipamentos monitorados e enviar essas informações ao servidor através da rede local de dados. Na Figura 2 encontra-se exemplificado a constituição da plataforma proposta.

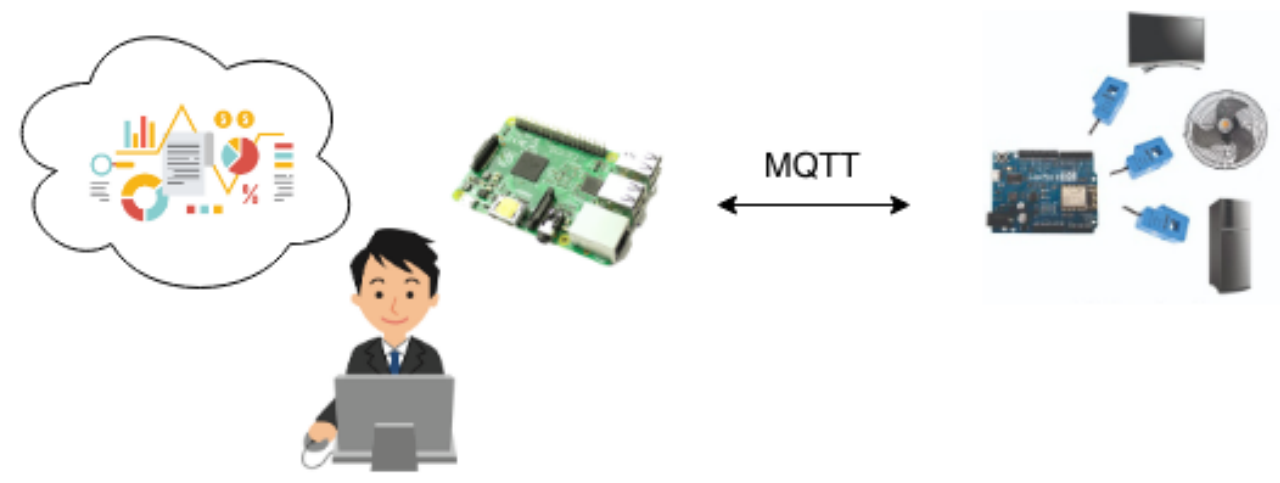

Figura 2. Esquemático da plataforma.

As informações enviadas ao servidor são armazenadas em um banco de dados e são apresentadas ao usuário através de uma interface web. $\mathrm{O}$ envio dos dados coletados 
pelo medidor desenvolvido é feito através do protocolo MQTT devido a sua versatilidade e performance em dispositivos embarcados. A comunicação acontece através de uma rede wireless, que facilita a implantação da plataforma com a minimização do uso de cabos na residência. Além disso, os dados enviados são encriptados através dos mecanismos de segurança suportados pelo protocolo MQTT, que é o caso da plataforma TLS (Transport Layer Security). Essa plataforma provê segurança na comunicação entre cliente e servidor, onde o principal objetivo é prevenir a interceptação e adulteração das mensagens [Dierks and Rescorla 2008].

\subsection{Dispositivo de Medição}

O hardware do sistema de medição é composto por dois elementos principais: uma placa Arduino e um sensor de corrente SCT-013. A placa Arduino utilizada é a Wemos, o microcontrolador desta é o módulo ESP8266EX. Esse módulo é capaz de prover comunicação sem fio através do padrão $802.11 \mathrm{~b} / \mathrm{g} / \mathrm{n}$, trazendo à plataforma Arduino um Wi-Fi nativo [Minatel 2016].

O programa que roda no Arduino tem como funções coletar as leituras de corrente enviadas pelos sensores, realizar o cálculo da potência e enviá-las a cada 30 segundos por um canal seguro para o broker MQTT que roda em uma Raspberry Pi. A Figura 3 traz um fluxograma com o funcionamento básico do programa.

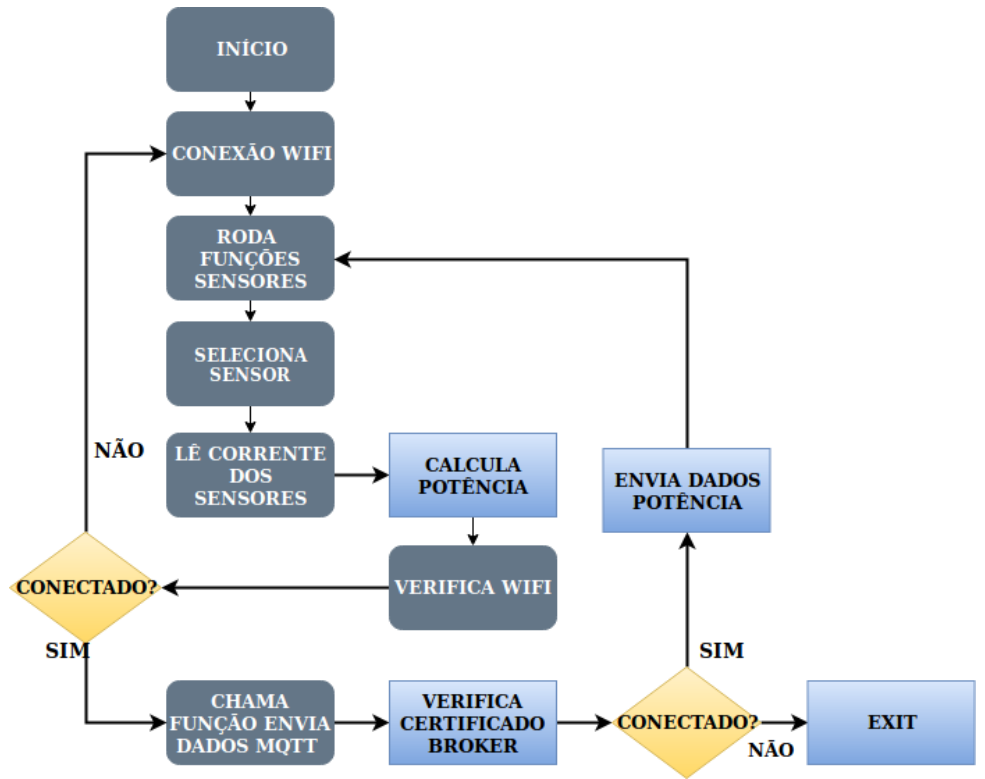

Figura 3. Fluxo de execução.

Como é possível observar no fluxograma, primeiramente é realizada a conexão à rede $W i-F i$ do usuário. Após é realizada a coleta dos dados de corrente de cada um dos sensores (SCT-013), assim a potência é calculada. Faz-se a verificação da conexão com a rede e os dados são enviados via MQTT ao servidor. Na próxima seção é tratado como a comunicação entre o dispositivo de medição e o servidor/broker MQTT funciona.

\subsection{Comunicação}

A comunicação do Arduino com o broker é feita de maneira segura, através da utilização da plataforma TLS. A principal função do broker é receber os dados enviados pelo Ar- 
duino e encaminhá-los ao destino. Para implementação da conexão segura é necessário que o broker possua um certificado digital, assim foi realizada a criação de uma entidade certificadora através da ferramenta OpenSSL e gerado o respectivo certificado.

\section{Sistema de Monitoramento}

O sistema de monitoramento da plataforma está hospedado em uma placa Raspberry Pi. O Raspberry Pi é um microcontrolador composto por um hardware único e de tamanho reduzido. Seu sistema operacional é uma versão GNU/LINUX. Na Raspberry Pi encontram-se hospedados o broker MQTT, o banco de dados da plataforma, além do servidor web responsável pela interface de monitoramento para o usuário.

O broker MQTT utilizado na plataforma é o Mosquitto. O Mosquitto é um broker open-source desenvolvido pela fundação Eclipse. As mensagens enviadas ao broker são salvas em um banco de dados SQL através de um script escrito na linguagem JavaScript. Esse script atua como um cliente MQTT que se inscreve em todos os tópicos existentes recebendo os dados de potência de todos os equipamentos monitorados e faz a inserção dos dados recebidos no banco de dados.

A interface de monitoramento da plataforma utiliza a linguagem de marcação HTML (Hypertext Markup Language), a API Google Chart para a geração dos gráficos, linguagem de programação PHP (Hypertext Preprocessor) para desenvolvimento da parte lógica da interface e roda em um servidor web Apache instalado na Raspberry Pi.

$\mathrm{Na}$ próxima seção são apresentados os testes e resultados obtidos com a implementação da plataforma.

\section{Testes e Resultados}

Para validar a plataforma desenvolvida foi elaborado um cenário de testes, que consiste no monitoramento de três equipamentos elétricos em uma residência: uma geladeira, um ventilador e uma televisão. A validação das medidas realizadas através do dispositivo de medição foi feita através da utilização de um alicate amperímetro que foi colocado no mesmo fio que o sensor SCT-013, ou seja, coleta as medidas de corrente do mesmo equipamento ao mesmo tempo.

O cenário de testes consistiu na coleta de 10 medidas de corrente consumida por uma televisão. Como a corrente da televisão é constante, o amperímetro nunca apresentou variação da medida. A Tabela 1 mostra os valores da medições feitas pelo amperímetro e pelo sensor.

O gráfico da Figura 4 mostra o desvio padrão da amostra de medidas de corrente realizadas pelo sensor. Quanto maior o desvio padrão, maior a dispersão dos dados medidos. Como é possível observar, a dispersão dos dados é bem pequena (0.019465068). Esta situação ocorre, principalmente, aos ruídos existentes no circuito do dispositivo de medição montado em uma protoboard com a utilização de inúmeros jumpers (fios). Uma solução para esse erro é utilizar um amplificador de sinal, que melhoraria a resolução da coleta de amostras feito pelo Arduino, além da construção do circuito do dispositivo um pouco mais profissional, em uma placa de circuito impresso.

A segurança das informações trafegadas entre o cliente Arduino e o broker Mosquitto foi implementada através da utilização da plataforma TLS com certificado digital 
Tabela 1. Dados de corrente coletados.

\begin{tabular}{cc}
\hline Amperímetro (exato) & Dispositivo de Medição (aproximado) \\
\hline $0,36 \mathrm{~A}$ & $0,47 \mathrm{~A}$ \\
$0,36 \mathrm{~A}$ & $0,45 \mathrm{~A}$ \\
$0,36 \mathrm{~A}$ & $0,45 \mathrm{~A}$ \\
$0,36 \mathrm{~A}$ & $0,45 \mathrm{~A}$ \\
$0,36 \mathrm{~A}$ & $0,42 \mathrm{~A}$ \\
$0,36 \mathrm{~A}$ & $0,42 \mathrm{~A}$ \\
$0,36 \mathrm{~A}$ & $0,47 \mathrm{~A}$ \\
$0,36 \mathrm{~A}$ & $0,47 \mathrm{~A}$ \\
$0,36 \mathrm{~A}$ & $0,46 \mathrm{~A}$ \\
$0,36 \mathrm{~A}$ & $0,47 \mathrm{~A}$ \\
\hline
\end{tabular}

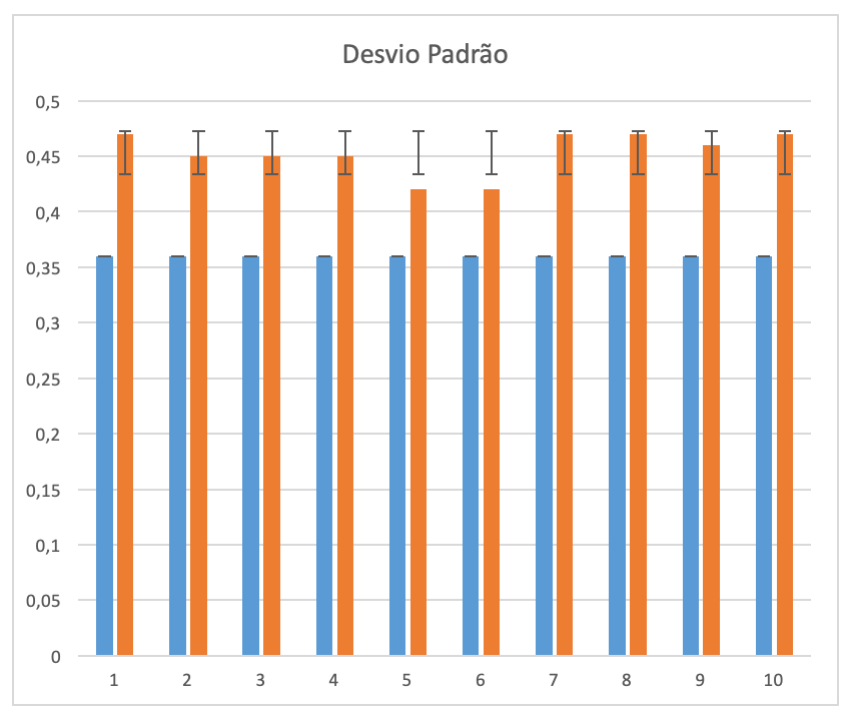

Figura 4. Gráfico do desvio padrão.

para autenticação, além de usuário e senha garantindo assim autenticação mútua. Para verificar a eficiência do método foi feita a captura de alguns pacotes da comunicação através do Wireshark e o resultado pode ser observado na Figura 5.

É possível observar que tanto o conteúdo da mensagem enviada quanto o tópico em que ela foi publicada, aparecem encriptados. Isso dificulta a ação de um indivíduo mal intencionado que tenha acesso à rede local do usuário, impossibilitando que o mesmo seja capaz de observar toda a troca de mensagens entre o dispositivo de medição e o servidor MQTT.

A interface de monitoramento da plataforma, consiste em uma página web hospedada localmente na rede do usuário. Entre as informações encontradas na página estão o consumo de energia em kWh em cada hora do dia, consumo total do dia e do mês corrente. Também são apresentadas informações desse mesmo consumo por equipamento, consumo diário e mensal. Além do consumo de energia, dados do valor estimado a ser pago pelo consumidor são mostrados ao usuário da plataforma. Esses valores são calcu- 


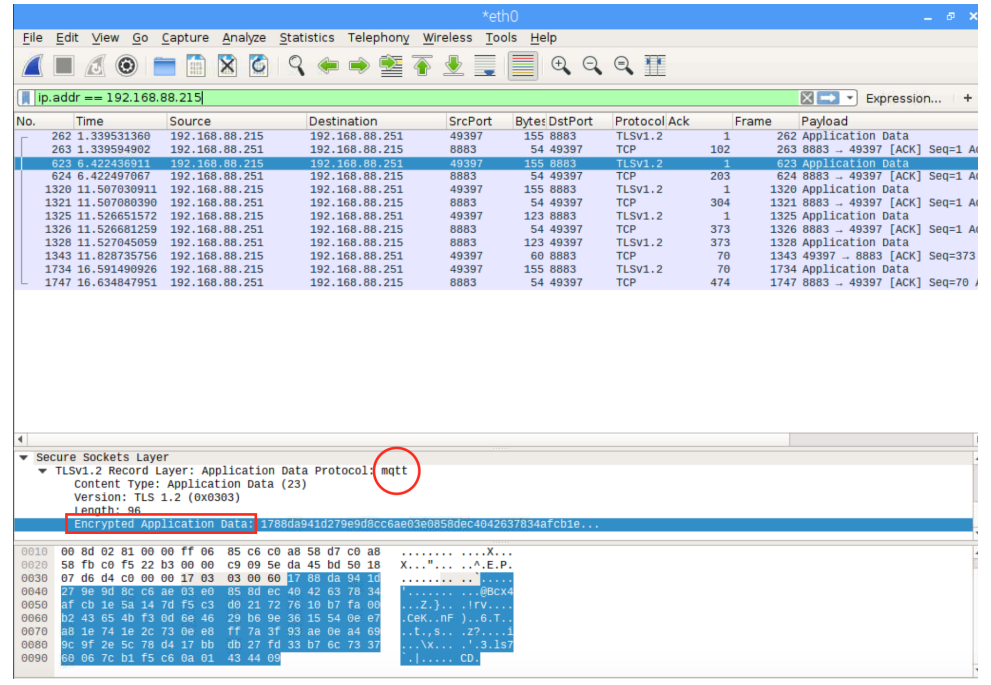

Figura 5. Captura Wireshark com TLS.

lados através de informações adquiridas através do usuário, como o valor do kWh da sua região.

O consumo horário do dia é apresentado ao usuário conforme Figura 6.

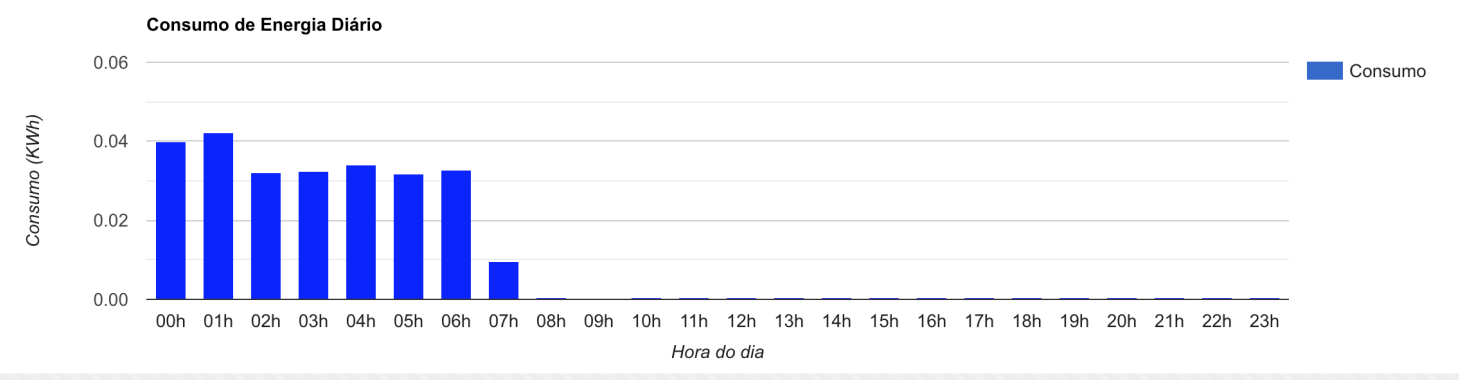

Figura 6. Consumo por hora do dia.

Os consumos individualizados de cada equipamento monitorado são apresentados em gráficos de pizza, conforme ilustrado na Figura 7.

Consumo Mensal de Energia por Equipamento (KWh)

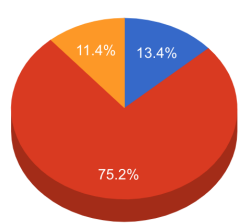

- Ventilador

Televisão

Figura 7. Consumo de energia por equipamento por mês. 
Por fim, a próxima seção deste trabalho apresenta as considerações finais e os trabalhos futuros a serem realizados.

\section{Conclusão e Trabalhos Futuros}

A plataforma desenvolvida neste trabalho busca trazer ao usuário um maior gerenciamento dos seus gastos com energia elétrica. De forma geral, a interface web da plataforma permite ao usuário visualizar o consumo individualizado de cada equipamento na sua residência, proporcionando um maior controle. A segurança dos dados trafegados na plataforma é um dos pontos mais importantes do trabalho apresentado. Garante ao usuário privacidade de seus dados e, também, a autenticação evita que novos dispositivos de medição sejam inseridos sem autorização ou que dados sejam manipulados. Outro ponto que merece destaque na plataforma desenvolvida é a possibilidade de monitoramento de vários equipamentos em um único dispositivo de medição, em uma única placa Arduino.

A plataforma abre espaço para inúmeras implementações futuras. Como o desenvolvimento de um aplicativo para dispositivos móveis, a geração de relatórios de consumo de cada mês, dia, por equipamento, entre outras possibilidades. Outra funcionalidade a ser incluída é ter uma página onde o usuário possa adicionar os impostos que têm incluídos em sua tarifa de energia. Além da inserção das bandeiras de energia, tornando o cálculo final mais aproximado do real a ser pago. Também futuramente pretende-se adicionar à plataforma uma interface para gerenciamento do usuário, onde o mesmo possa interagir com os equipamentos monitorados. Através da definição de horários para que determinados equipamentos sejam desligados e alertas quando o consumo chegou a um valor pré-definido.

\section{Referências}

CGEE (2012). Redes elétricas inteligentes: contexto naional. http://www.cgee.org.br/atividades/redirect/8050. Acessado em Outubro/2017.

Dierks, T. and Rescorla, E. (2008). The transport layer security (tls) protocol. https://www.ietf.org/rfc/rfc5246.txt. Acessado em Dezembro/2018.

Minatel, P. (2016). Wemos d1 - o esp8266 com cara de arduino. http://pedrominatel.com.br/pt/esp8266/wemos-d1-o-esp8266-com-cara-de-arduino/. Acessado em Dezembro/2018.

Yan, Y., Qian, Y., Sharif, H., and Tipper, D. (2013). A survey on smart grid communication infrastructures: Motivations, requirements and challenges. IEEE COMMUNICATIONS SURVEYS TUTORIALS, 15(1):15-22.

Yuan, M. (2017). Conhecendo o mqtt. https://www.ibm.com/developerworks/br/library/iotmqtt-why-good-for-iot/iot-mqtt-why-good-for-iot-pdf.pdf. Acessado em Novembro/2018.

Zhou, X., Ma, Y., Gao, Z., and Wang, H. (2017). Summary of smart metering and smart grid communication. In Proceedings of IEEE INTERNATIONAL CONFERENCE ON MECHATRONICS AND AUTOMATION, Takamatsu, Japan. IEEE. 